\title{
Keeping an eye on the human-animal interface
}

\author{
A look at viral surveillance and how researchers and veterinarians identify what animals may be susceptible to \\ different viruses, including SARS-CoV-2.
}

\section{Ellen P. Neff}

A s the COVID-19 pandemic continues, the problem lies with people. Human cases abound, regardless of where else the SARS-CoV-2 virus might be found. But as reports of positive cases in different species continue to pop up, there are nevertheless important concerns about transmission from the humans hosting the virus into other animals. That's not entirely unexpected, says Kaitlin Sawatzki, a postdoctoral researcher at Tufts University who studies zoonotic diseases. "We know coronaviruses are zoonotic, and we know that they are especially good at jumping between different species - it's kind of their superpower."

Since last spring, Sawatzki has been helping to coordinate an ongoing community science project called CoVERS, which is surveilling owners and animals for evidence of SARS-CoV-2 transmission. She and her colleagues were initially concerned for pets, imagining for example that dogs licking their owners' faces would turn up left and right with COVID-19. "But then we started to see these unusual events," she says, such as tigers in zoos testing positive. "I don't think tigers are licking their zookeepers' faces, and so that really opens up the question: is there a bigger problem with incidental human transmission to other animals - not just your dog or your cat, but maybe to wildlife?" she says.

In the grand scheme of things, viruses that can make the jump from one species to another - and onward - remain rare. "Most viruses are species-specific," says Sarah Caddy, a veterinarian who studies the immunology of viral infections at the University of Cambridge and early on in the COVID-19 pandemic ran a small research project to screen pets in the UK for the virus. For virus spillover to occur, there first needs to be sufficient interspecies contact. The virus has to bind to a potential new host's cells, get in, and replicate, all while evading the host's immune system. It then has to make it out again in sufficient quantity to cause additional infections.

But spillover does happen - as the current pandemic attests - and the

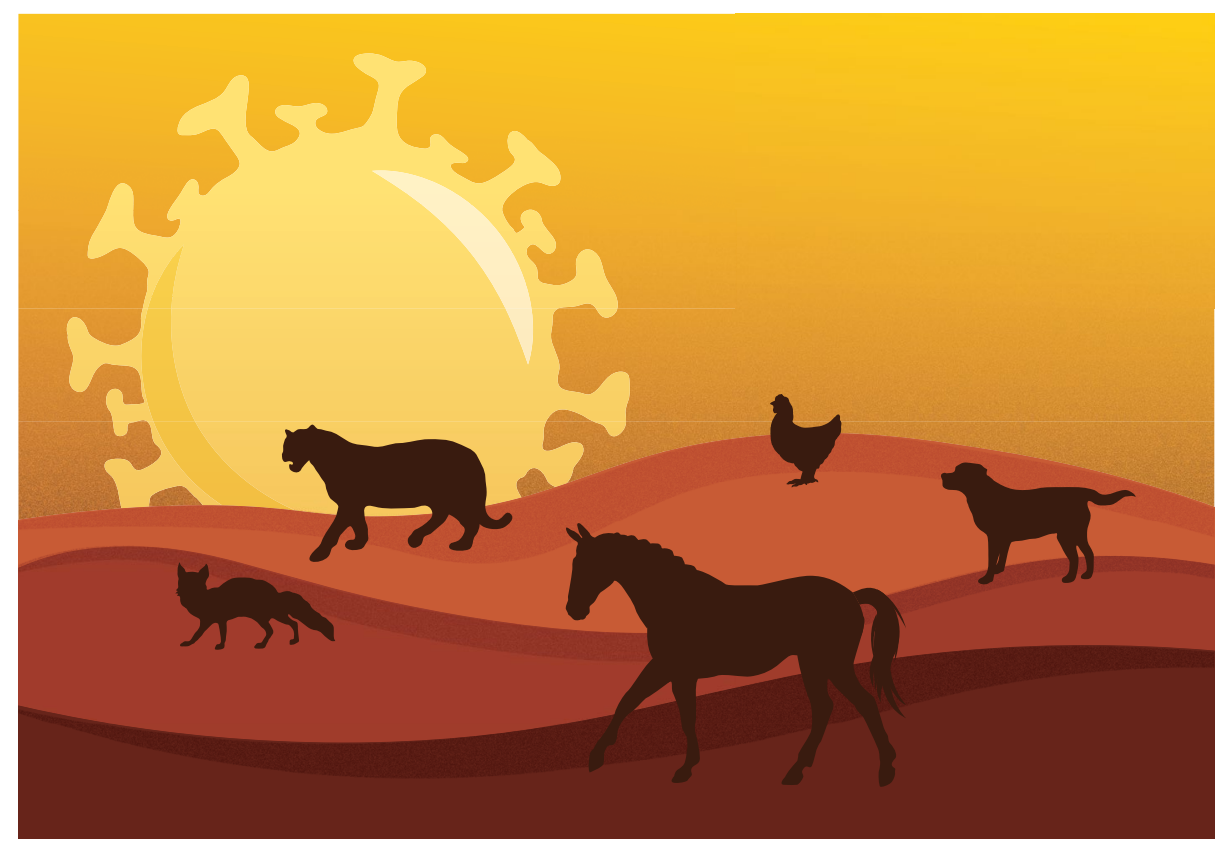

What's on the horizon? For SARS-CoV-2 and beyond, research is underway to determine what animals might be at risk. Credit: Marina Spence / Springer Nature

interfaces between human and animals continue to shift and change, bringing novel combinations of species and viruses into contact. When a threat emerges, how exactly do researchers figure out what animals might be at risk? Answering that question involves collaborations across both skillsets and scales.

\section{Cause for concern?}

There are a lot of animals, and viruses, out there, and researchers need to prioritize what ones may be cause for concern. Sometimes, symptoms of a disease are obvious - think of the mass mortality events observed in crows infected with West Nile Virus (a mosquito-borne virus that can also cause febrile illness in humans), says Angela Bosco-Lauth, a virologist and veterinarian and at Colorado State University. Often, signs are much subtler - if they are there at all.

Animals in close contact with people are therefore a common starting point.
Pets fall into that category, especially those whose owners have come down with a viral infection, as do agricultural animals, which are often raised at high densities that can encourage viral spread, along with wildlife that live near human populations - or are having their own territories encroached upon.

Susceptibility tends to follow patterns, which can also help inform what animals may need closer looks. "I start with what is known about a class of pathogens," Bosco-Lauth says. "When it comes to the animals, that gets trickier because they all respond differently, but we usually can use past experience with related pathogens to look at what we might expect." For coronaviruses, for example, it's known from prior outbreaks that mustelids - ferrets, minks, even skunks - can become infected, as can cats, while influenzas tend to hit birds. Transmission method matters too: mosquito-borne viruses tend have a wider range of potential hosts than airborne ones. 


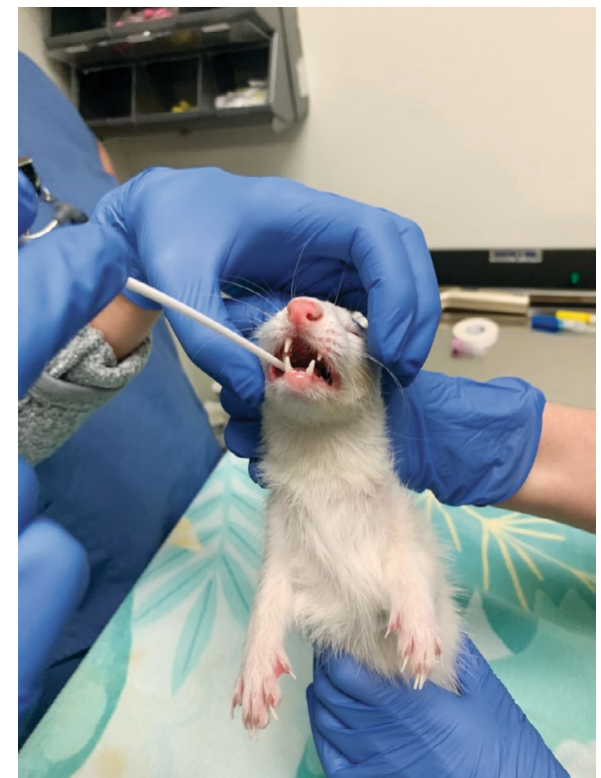

Open wide! Clinical veterinary staff at Foster Hospital for Small Animals at Cummings Veterinary Medical Center at Tufts University perform a mouth swab on a ferret, with permission from its owner, to obtain a sample for the CoVERS study. Credit: Tufts University

The process for screening a particular animal for a particular virus is similar to that for humans. Blood samples can be taken, byproducts of clinical exams or drawn from wildlife or agricultural species to look for antibody responses via serological tests. "But my caveat with that is a serological response only means that an animal has been exposed, and doesn't indicate anything about their susceptibility to infection," says Bosco-Lauth.

It's also possible to test for genetic evidence of the virus itself, via nasal swabs or samples of saliva or stool (depending on the virus in question). "At the end of the day, the qPCR tests are identical," says Caddy. But, she notes, "it's one thing to say, yes the tiny qPCR chunk - about 100 base pairs is present in a dog, but it's another to say actually the entire virus genome is present in a dog and it's identical to the virus genome in a human." Whole genome sequencing of samples can confirm a more direct link between a person and an animal.

For screening, getting samples can be the challenge, says Caddy. During her PhD research on human norovirus in dogs, she was still working as a veterinarian and was able to collect her own stool samples; for studying SARS-CoV-2, she's relied on active veterinarians to collect those nasal swabs from their patients, underscoring the need for infectious disease researchers to establish networks with different parties that contribute to disease monitoring. For domesticated animals, that includes veterinarians and owners; for wildlife, researchers work with government agencies to obtain the the appropriate permits and for help with collection.

But what about those animals that are a little trickier to collect samples from - rare or hard to find wild animals, or even those domesticated ones that just aren't suited to swabbing?

As Jacquelyn Brown saw reports about pet cats and dogs testing positive for SARS-CoV-2, she started to wonder about her horse. "We handle horses' faces so much...There's a lot of contact between our hands and their respiratory system," says Brown, a researcher at Vanderbilt University who has, among other viruses, studied equine encephalitis - a pathogen that can cross between humans and horses. But the simple, swab-based tests to screen other animals for SARS-CoV-2 didn't apply to the larger equines. "You can't do a nasal or throat swab in a horse without sedating them, because the distance you have to go is measured in many, many inches," Brown says.

In silico modeling can help. As viral genomes are sequenced and virologists and structural biologists unravel how viral proteins interact with host cells to gain entry, modeling can identity animals that have genetic and structural similarities to those known to be susceptible to a virus. For SARS-CoV-2 and a prior coronavirus that caused an outbreak of severe acute respiratory syndrome in 2003, the human protein angiotensin I converting enzyme 2 (ACE2) is considered the most likely receptor by which the virus infiltrates host cells. With that knowledge, Brown and collaborators at Vanderbilt that included virologists, immunologists, and structural biologists analyzed the genetic and structural differences of the ACE2 protein between animals that had already been directly tested for SARS-CoV-2 susceptibility, such as mice, ferrets, and macaques, among others; the team then developed a predictive model ${ }^{1}$ (horses ended up with high prediction scores for susceptibility), along with an accompanying web tool that others can use with their own species of interest. At the time they were preparing their publication, the ACE2 sequence for minks, farms of which have experienced SARS-CoV-2 outbreaks, was not yet available, notes senior author Meena Madhur; when that data was uploaded to the NCBI genome repository, the team ran it through the tool. "Minks showed up around where the ferrets were," she says.

Other groups have done similar genetic and structural analyses for SARS-CoV-2, including work from researchers at the University of California, Davis that analyzed 410 different vertebrate animals ${ }^{2}$. With enough genetic and structural knowledge, similar approaches can be applied with other viruses as well. Predicted susceptibility, however, doesn't mean that that animal will necessarily get sick, or that the virus will be able to replicate inside of it sufficiently to be passed on to another animal. "It's a low pass filter of, 'do I move forward?", says Brown, one that can, for example, help researchers prioritize what cell lines to study. It can also be a hint that species with high predicted susceptibility might merit some additional monitoring. Serology results and genomic evidence come with similar caveats - the virus may have been in that animal, but you can't say that the animal became sick or began shedding it.

Working in vitro with cell lines from various animals can build on initial suspicions. "That's the first place I want to go, to see whether or not the individual cells in the host are capable of supporting infection," says Tufts virologist Wendy Puryear. In cell lines, researchers can look more closely at details such as viral binding and growth kinetics, with added elements of the host immune system or other genetic details, such as co-receptors, at play that modeling may lack or overlook.

Having proper partnerships is key for getting those samples needed to create those cultures (for nontraditional species without commercially available toolkits, it's not always easy to get cell lines up and running). For Puryear and her colleagues, that's included veterinarians, staff in the National Park Service and other government agencies, and wildlife rehabilitators, each of whom can provide the necessary samples from recently deceased or euthanized animals (unrelated to the virus intended to be studied).

Screening, modeling details such as receptor homology, and in vitro work in cell lines are important proxies for infections, says Anna Fagre, a veterinarian working on her $\mathrm{PhD}$ at Colorado State who studies viral ecology in wildlife, including bats and deer mice. "You owe it to the animals to do your due diligence," she says, before considering experimental infections.

Experimental infections involve direct viral inoculation, attempting, as is possible, to mimic the likely viral route of entry - or example, injections for arthropod-borne diseases, inhalation for air-borne ones. Researchers consider pathological responses and whether live virus can be retrieved from the animal. "Then we look at, can they transmit?" says Bosco-Lauth. How long is an animal infectious for, and what are the conditions that enable transmission to other 
members of the same species - and then across to others? If a virus does make it into a different species, does its genetic sequence change along the way?

For facilities capable of conducting experimental infections, a limiting factor is whether the species that researchers want to study can be trapped and/or imported, says Bosco-Lauth. For example, Chinese horseshoe bats - a suspected natural host of SARS-CoV-2, can't currently be imported into the United States, while opportunities to trap native relates is limited, given the disease risk humans currently pose to the bats.

Model species can be used, but compatibility must be justified and taxonomic differences that may influence host-virus co-evolution need to be taken into account, says Fagre. She has for example worked with an experimental colony of Jamaican fruit bats to study viral pathogenesis, noting that these are New World bats: viruses studied in them may potentially behave differently than in natural hosts found elsewhere in the world, she says.

Conducting experimental work, particularly in larger species, takes special biocontainment facilities and expertise in handling less traditional lab animals. When the link was proposed during an outbreak of Middle Eastern Respiratory Virus (MERs) between the coronavirus MERS-CoV and camels, the NIH National Institute of Allergy and Infectious Diseases (NIAID) facility in Montana brought in dromedary camels. "They're definitely not your run-of-the-mill laboratory animals, and a lot more planning has to go into it," says NIAID researcher and veterinarian Danielle Adney. Animal crackers became a favorite for motivating the camels; follow up work has continued in related, though ever so slightly smaller, camelid relatives: alpacas.

A lot of time is spent figuring how to get the most out of the animals with what's available, and deciding how to interpret clinical results in animals that often don't have baseline information available. "If we don't know normal, how can we assess abnormal?" says Fagre, emphasizing the need to better understand the basic biology and life histories of a wider range of animals.

Resources for working with nontraditional species can also be limited, even if the animals themselves are available. "There's not usually reagents available," says Adney. For example, antibodies are needed for immunology studies; these are readily available for mice and more traditional species, but less so for the less familiar animals out there. “There's nothing special about a mouse - it's just that people have put a lot of money and effort into it," says Adney's colleague Neeltje van Doremalen, who usually works with those more traditional models to study viral transmission and the development of vaccines. Even resources for those animals that are becoming more widely used, such as hamsters for studying coronaviruses, can be still be wanting relative to those for their murine cousins, she says.

\section{Then what?}

The results of lab-based experimental infections still come with caveats too. "It's very difficult to simulate what's happening out in the real world in the lab setting," says Bosco-Lauth. "You don't get the gamut of what's seen in nature - or even in the grocery store."

Sometimes a clear transmission event in the lab will fail to materialize in life - or vice versa. "That ends up leading to a lot more refining of our studies," says Bosco-Lauth. For example, both cats and ferrets are highly susceptible to SARS-CoV-2 in the lab - the latter has become an important animal model - but transmission events in real-life homes have been much more mixed. For example, consider the case of COVID-19-positive owners living with 29 pet ferrets ${ }^{3}$. "I was absolutely sure that every single ferret was going to be infected," says Sawatzki. But none were, nor did antibodies turn up in the animals. "That really got us thinking about what is the difference between a lab-infected animal and a naturally infected animal," she says. "The world is messy."

In homes, transmission isn't forced, so researchers need to consider how closely animals are actually interacting, and whether there are variables such as age or sex at play. "It ends up going back to a little more of an epidemiology study then - trying to figure out what's happening outside that we aren't able to replicate, and then how can we fine-tune what we do in the lab," says Bosco-Lauth.

Regardless of how imperfect or incomplete the data may be, researchers and public health stakeholders do need to decide what to do with the information that lab studies reveal "My first concern is usually animal health," says Bosco-Lauth, noting the need to consider all the scales, from an individual animal to its particular population to the species level. Understanding interactions are key - a social animal is much more likely to spread a disease, while more solitary creatures are likely to be 'dead end' hosts.

Vaccines can be considered - such an effort was undertaken for the endangered black footed ferret in the US over the summer - but debate about such efforts remains; human vaccination programs, at least for COVID-19, are the pressing priority. To many involved, keeping animals protected comes down to human behavior and preventing spread in the first place. "Usually, the advice is leave the animals alone, but you can't do that anymore," says Nichola Hill, a disease ecologist at Tufts. "We're in this era where we just have such a huge human footprint. We have to figure out where that sweet spot is that gives animals enough space to do what they do and gives humans a livelihood and the ability to carry on with their lives."

Between human encroachment - the cutting down of forests and building of roads and expansion urban areas - and climate change, food webs are being disrupted, new environmental stressors are being introduced, and species ranges are shifting. "All of these things impact the health of the animal and the susceptibility of the animal to the disease and now, these animals are interacting with new animals that they hadn't been before," says Puryear. Consider Phocine Distemper virus, a morbillivirus that infects seals (a more familiar relative in humans is the measles virus). Harp seals, native to the arctic, appear to get on just fine with the virus; but as they descend to sub-polar waters in search of shifting populations of declining prey, they encounter their harbor seal cousins, which lack immunity to Phocine Distemper - past outbreaks have caused mass mortality events in harbor seal populations over a span of just a few months. "How these things cross species is a very big concern," says Puryear.

That means bringing the bigger, "messier" ecological picture into the frame, says Hill. Researchers need to collect large-scale data about human and animal

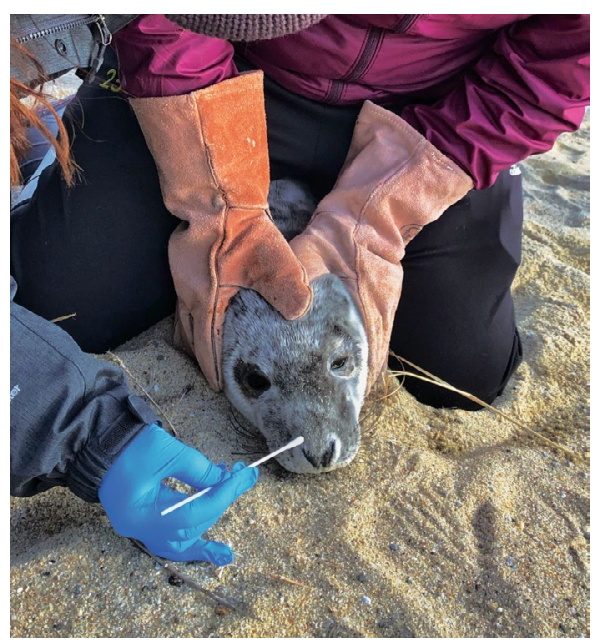

From the field | A weaned grey seal on an island off Cape Cod is sampled for respiratory viruses. Image collected under NMSF permit 17670. 


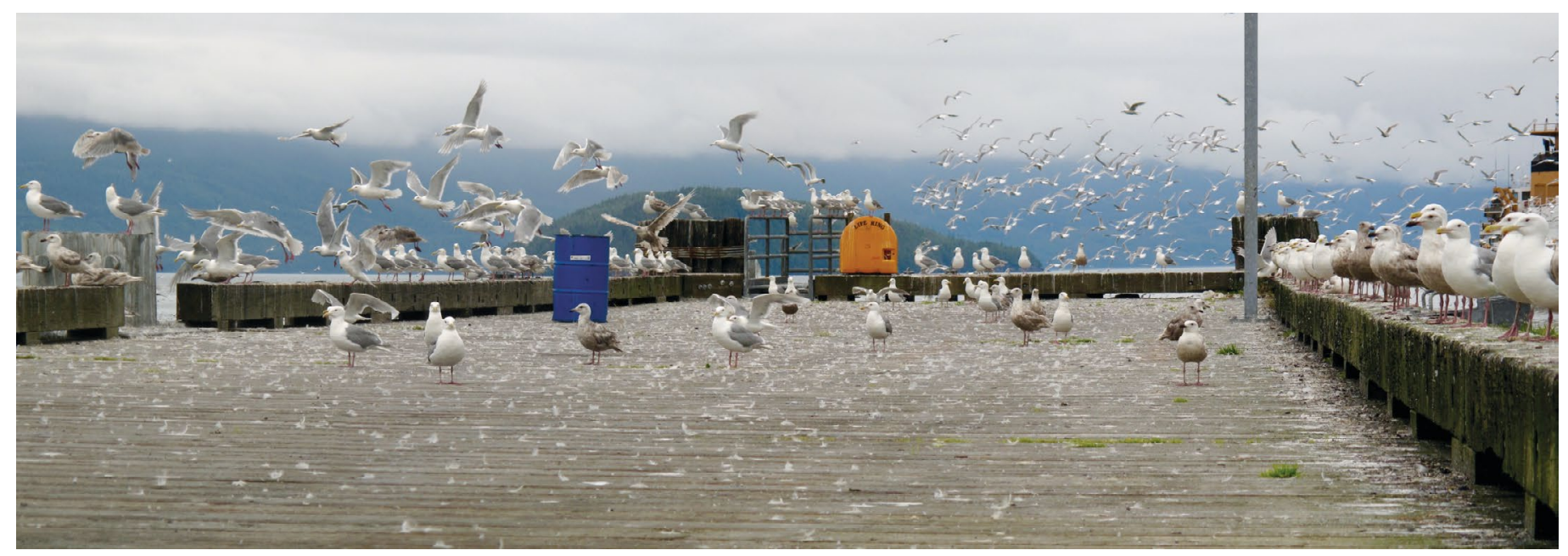

At the interface | Gulls tend to turn up around fishing docks, such as this one near a cannery in Alaska. Nichola Hill has been leading an ongoing avian influenza surveillance project there, the results of which can help inform what steps humans might take to minimize those aggregations, such as improving waste disposal. Credit: Nichola Hill

movement and interactions and how ecosystems are changing. "Ecological stress; habitats; the climate crisis - how does that jive with host jumping, and more of the molecular characteristics of the virus?", says Hill. Work should go in both directions, she says, "where what we observe in nature gives us hypotheses to test in a reductionist fashion in the lab. I do think they can close the loop for each other."

The time to do this work is before outbreaks occur. "Genetic sequencing has never been cheaper or more accessible. You can take that genetic sequence; we can clone things in the lab. We can test viruses, or even just parts of viruses, to see if something is maybe a risk for human infection. This can all happen before any transmission happens," says Sawatzki. Paired with ecological data, the genetic sequences of viruses can help tease apart viral evolution and where interspecies interactions are occurring; whether particular mutations are shared - or notably different - between susceptible animals can help inform how a virus might spread.

Continued surveillance at high-risk human-animal interfaces will be key. "As people continue to interface with wild animals and our interactions with animals change, I think we are going to continue to see spillover events," says Adney, noting the need to understand the risks in order to keep both humans and animal populations safe. "We still need a better idea of what's out there," she says.

There are a number of surveillance projects around the world aiming to do just that, such as the newly funded Strategies to Prevent Spillover and for the past 11 years, PREDICT, both supported by the US Agency for International Development's Emerging Pandemic Threats program. Country teams collaborating through PREDICT have been collecting data and testing for viruses in areas considered high-risk for viral spillover due to close contact between people and animals; to date, they've sampled over 164,000 animals and people, identifying 949 novel viruses and 217 known ones in those samples. Viruses of concern are prioritized for viral characterization studies to determine if they do in fact have zoonotic potential, says Tracey Goldstein, the Co-Principle investigator and Pathogen Detection lead for the PREDICT project at the University of California, Davis One Health Institute.

For example, when PREDICT identified a novel MERS-CoV relative in bats found in homes in Uganda, the teams involved sequenced the full genome of that virus and started in vitro work with different receptor constructs to see if infections of human cells was possible. Based on that data, they determined that this particular virus was not likely to be a human pathogen, but the work provides insight into what might need to change for it to become pathogenic, says Goldstein.

As more and more viruses are better described and characterized, patterns will likely start to emerge, says Goldstein. Those patterns may not enable researchers to pinpoint exactly where and when a particular spillover event will occur, but a better understanding of the characteristics of viruses that can jump between species, the animal hosts those viruses potentially can infect, and where viruses, animal hosts, and humans occur together should help stakeholders focus efforts to prevent outbreaks in the first place, she says, such as putting together education campaigns about living safely with bats.

While humans are indeed at risk from zoonotic diseases, it's important to remember that we bring that risk upon ourselves. "The human-animal interface is tremendous," says Bosco-Lauth. "Most of these interactions would never happen if it weren't for humans. We are the ones driving them, and so it's up to us to manage that and to not cause more harm than good. We don't ever want to condemn an animal because maybe that species is the cause of a disease - it's because we interface with them that we're now getting it," she says. "It's up to us to protect our animals as much as ourselves from zoonotic infections, and we owe to the animals to be stewards of their health."

\section{Ellen P. Neff $\bowtie$ \\ Lab Animal. \\ $\bigotimes_{\text {e-mail:ellen.neff@us.nature.com }}$}

Published online: 18 February 2021

https://doi.org/10.1038/s41684-021-00725-y

References

1. Alexander, M. R. et al. FASEB J. 34, 15946-15960, https://doi. org/10.1096/f.202001808R (2020).

2. Damas, J. et al. PNAS 117, 22311-22322, https://doi.org/10.1073/ pnas.2010146117 (2020).

3. Sawatzki, K. et al. bioRxiv https://doi. org/10.1101/2020.08.21.254995 (2020). 